Article

\title{
Synthesis, Insecticidal, Fungicidal Activities and Structure-Activity Relationships of Tschimganin Analogs
}

\author{
Yueting Zhou ${ }^{1}$, Chunjuan Wang ${ }^{1}$, Fang Xin ${ }^{1}$, Xiaoqiang Han ${ }^{1, *}{ }^{\mathbb{C}}$, Jie Zhang ${ }^{2, *}$ and Ke Sun ${ }^{3}$ \\ 1 Key Laboratory at Universities of Xinjiang Uygur Autonomous Region for Oasis Agricultural Pest \\ Management and Plant Protection Resource Utilization, College of Agricultural, Shihezi University, \\ 221st Beisi Road, Shihezi 832002, China; zhouyueting@stu.shzu.edu.cn (Y.Z.); \\ wchunjuan@126.com (C.W.); xinf@stu.shzu.edu.cn (F.X.) \\ 2 Key Laboratory for Green Processing of Chemical Engineering of Xinjiang Production and Construction \\ Group, School of Chemistry and Chemical Engineering, 221st Beisi Road, Shihezi 832003, China \\ 3 State Key Laboratory of the Discovery and Development of Novel Pesticide, \\ Shenyang Sinochem Agrochemicals R\&D Co., Ltd., No. 8-1 Shenliao Dong Road, \\ Tiexi District, Shenyang 110021, China; sunke@sinochem.com \\ * Correspondence: hanshz@shzu.edu.cn (X.H.); zhangjie-xj@163.com (J.Z.); Tel.: +86-993-205-8060 (X.H.); \\ +86-993-205-7215 (J.Z.)
}

Received: 15 May 2018; Accepted: 16 June 2018; Published: 18 June 2018

\begin{abstract}
For the first time, a novel series of tschimganin analogs were designed, synthesized, and evaluated for their insecticidal and fungicidal activities. Their structures were characterized by ${ }^{1} \mathrm{H}-\mathrm{NMR},{ }^{13} \mathrm{C}-\mathrm{NMR}$ and HRMS. Some of these compounds displayed excellent insecticidal and fungicidal activities, suggesting that they have potential to be used as bifunctional agrochemicals. Compound $\mathbf{3 d}$ and $3 \mathbf{g}$ with electron donating groups showed better inhibitory activity and growth inhibition activity towards Helicoverpa armigera (Hübner). The properties and positions of the substituents on the benzene ring have an important influence on the acaricidal activity of tschimganin analogs. Topomer comparative molecular field analysis (CoMFA) was employed to develop a three-dimensional quantitative structure-activity relationship model for the compounds against Tetranychus turkestani Ugarov et Nikolski. It was indicated that higher electronegativity was beneficial for acaricidal activity. Moreover, compound 3r having a 2-hydroxy-3,5- dinitrophenyl moiety displayed a fungicidal spectrum as broad as azoxystrobin against these phytopathogens.
\end{abstract}

Keywords: tschimganin analogs; insecticidal activities; fungicidal activity; structure-activity relationship (SAR)

\section{Introduction}

Botanical pesticides refer to plants, crude plant extracts or the derivatives of the active ingredients used for the protection of crops and stored products from insect pests. These can be recommended as an ecochemical and sustainable strategy for the management of agricultural pests due to their biodegradable nature, systemicity after application, capacity to alter the behavior of target pests, and their favorable safety profile [1]. The exclusively Old-World genus Ferula belongs to the family Umbelliferae, with about 150 species distributed throughout the Mediterranean area and central Asia, especially in the former USSR, Iran and northwest China (e.g., Xinjiang Province) [2]. Some plants of the genus Ferula have been used as pharmaceutical plants in many countries for centuries [3]. Ferula is a rich source of biologically active compounds, such as coumarin derivatives, sesquiterpenes, aromatic lactones, and disulfide compounds [4]. Plant extracts and 
metabolites from the Ferula genus were reported to possess many biological features, including antiviral [5], anti-inflammatory [6], antitumor [7,8], anticancer [9], antiulcerative [10], antidiabetic [11], antibacterial [12,13], acaricidal [14], antiprotozoal, and antiglycation [15] activities. The diastereomeric monoterpene benzoates tschimganin (1) and isotschimganin (2) were isolated from Ferula dissecta (Figure 1). The growth inhibition effects of $\mathbf{1}$ and $\mathbf{2}$ exhibited different degrees of cytotoxic effects against HeLa cells at $24 \mathrm{~h}$ after treatment with $\mathrm{IC}_{50}$ values of 32.1 and $29.1 \mu \mathrm{M}$, respectively [16]. Tschimganin (1) was also found to be a novel drug resistance reversal agent for Staphylococcus aureus [17]. The pooled results indicate that tschimganin (1) has good antibacterial and anti-tumor activities. However, the synthesis and structure-activity relationship of tschimganin analogs have not been reported. Inspired by these results, we were encouraged to further investigate the effect on fungicidal and insecticidal activities by tschimganin. Therefore, in this report, we systematically designed and synthesized a novel series of tschimganin analogs (3) and evaluated them for insecticidal and fungicidal activities. Their structure-activity relationship (SAR) also was studied.

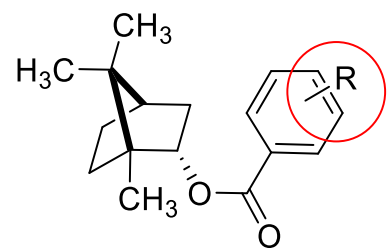

3

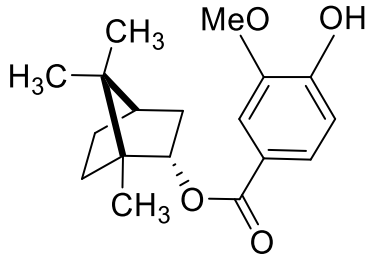

1

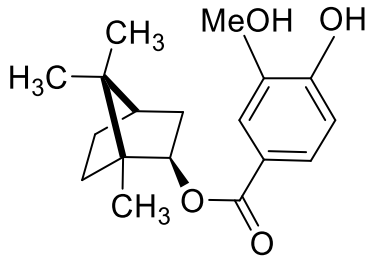

2

Figure 1. Design strategy for novel tertiary alcohols.

\section{Results and Discussion}

The synthesis of tschimganin analogs 3, which features a catalytic esterification, is shown in Scheme 1. The tschimganin analogs 3 were prepared in excellent yield from commercially available substituted benzoic acids 4 and $1 S, 2 S, 4 R$-fenchyl alcohol (5) via room temperature esterification catalyzed by 4-dimethylaminopyridine (DMAP) and dicyclohexylcarbodiimide (DCC).

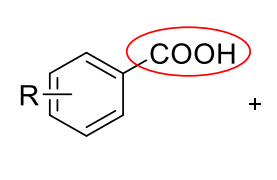

4

3a $\quad \mathrm{R}=\mathrm{H}$

3b $\mathrm{R}=2-\mathrm{CH}_{3}$

3c $\mathrm{R}=4-\mathrm{CH}_{3}$

$3 \mathrm{~d} \quad \mathrm{R}=2-\mathrm{OMe}$

3e $\mathrm{R}=3-\mathrm{OMe}$

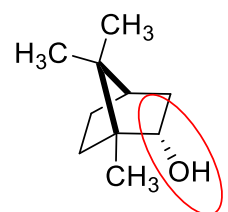

5

3f $\mathrm{R}=4-\mathrm{OMe}$

$3 \mathrm{~g} \quad \mathrm{R}=4-t$ - $\mathrm{Bu}$

3h $\mathrm{R}=2-\mathrm{NO}_{2}$

3i $\mathrm{R}=3-\mathrm{NO}_{2}$

3j $\mathrm{R}=4-\mathrm{NO}_{2}$



$3 \mathrm{k} \quad \mathrm{R}=2-\mathrm{F}$

$31 \mathrm{R}=3-\mathrm{F}$

$3 \mathrm{~m} \mathrm{R}=4-\mathrm{F}$

3n $\mathrm{R}=2-\mathrm{Cl}$

3o $\mathrm{R}=3-\mathrm{Cl}$
$3 \mathrm{p} \quad \mathrm{R}=4-\mathrm{Br}$

3q $\quad \mathrm{R}=4-\mathrm{I}$

$3 \mathrm{r} \quad \mathrm{R}=2-\mathrm{OH}-3,5-$ dinitro

3s $\mathrm{R}=4-\mathrm{CN}$

3t $\mathrm{R}=1$-naphthylacetic acid

Scheme 1. Synthetic route for tschimganin analogs 3.

To characterize the biological potential of these tschimganin analogs 3, the insecticidal activities of all the new compounds were tested against Helicoverpa armigera (Hübner), Tetranychus truncates and Tetranychus turkestani Ugarov et Nikolski, and their in vitro antifungal activities were evaluated against seven plant pathogenic fungi. The commercial insecticides chlorantraniliprole, biflenazate, pyridaben, hexythiazox and commercial fungicides azoxystrobin, kresoxim-methyl, trifloxystrobin were used as control. As shown in Table 1, the tschimganin analogs 3 exhibited moderate or weak activity to H. armigera (Hübner). Only the compounds $\mathbf{3 d}, \mathbf{3 e}, \mathbf{3 f}$, and $\mathbf{3 g}$ containing electron-donating groups showed medium insecticidal activity. Compound $3 \mathrm{~g}$ showed the best activity $(60 \%)$, which implied that 
the electron-donating groups (alkyl and alkoxy) were beneficial to the insecticidal activity. Interestingly, most of the tschimganin analogs displayed H. armigera (Hübner) growth inhibition activity. Therefore, we further studied their effect on the metabolic enzyme activity of bollworm (Figure 2).

The target compounds showed different inhibitory activities on cotton bollworm metabolic enzymes. Compound $\mathbf{3 d}$ and lufenuron showed similar effects on the six enzyme activities: they displayed no activity on chitinase, inhibition of carboxylesterase (CarE) and polyphenol oxidase (PPO), and activation of glutathione S-transferase (GST) and phosphatases (acid phosphatase, ACP and alkaline phosphatase, AKP). Compound $\mathbf{3 g}$ and chlorantraniliprole showed significant activation of PPO activity, and no effects on GST activity. In contrast $\mathbf{3 g}$ displayed a slight inhibition of chitinase while chlorantraniliprole showed activation of chitinase. Meanwhile, chlorantraniliprole displayed a significant inhibition of phosphatases (ACP and AKP). Inspired by the tschimganin analogs $H$. armigera (Hübner) activity, we were encouraged to further investigate the effects on the cotton spider mite.

Table 1. In vitro inhibitory activity against the cotton bollworm and the two cotton spider mite species at $50 \mu \mathrm{g} / \mathrm{mL}^{\mathrm{a}, \mathrm{b}}$.

\begin{tabular}{|c|c|c|c|c|c|c|}
\hline \multirow[b]{2}{*}{ Compound } & \multicolumn{2}{|c|}{ H. armigera (Hübner) } & \multicolumn{2}{|c|}{ T. truncatus } & \multicolumn{2}{|c|}{ T. turkestani } \\
\hline & $\begin{array}{c}\text { Mortality } \\
\text { Rate \% }(48 \mathrm{~h})\end{array}$ & $\begin{array}{l}\text { Growth and } \\
\text { Development }\end{array}$ & $\begin{array}{c}\text { Mortality } \\
\text { Rate \% }(24 \mathrm{~h})\end{array}$ & $\begin{array}{c}\text { Mortality } \\
\text { Rate } \%(48 \mathrm{~h})\end{array}$ & $\begin{array}{c}\text { Mortality } \\
\text { Rate \% }(24 \mathrm{~h})\end{array}$ & $\begin{array}{c}\text { Mortality } \\
\text { Rate \% }(48 \mathrm{~h})\end{array}$ \\
\hline $3 a$ & 0 & ND & 15.56 & 63.33 & 10.41 & 40.62 \\
\hline $3 b$ & 3 & dysplasia & 13.33 & 48.89 & 8.93 & 50.00 \\
\hline $3 c$ & 10 & dysplasia & 27.78 & 45.56 & 22.29 & 46.87 \\
\hline $3 d$ & 37 & dysplasia & 55.56 & 83.33 & 26.75 & 35.94 \\
\hline $3 e$ & 30 & dysplasia & 30.00 & 40.00 & 25.27 & 71.87 \\
\hline $3 \mathrm{f}$ & 23 & dysplasia & 38.89 & 52.22 & 47.57 & 70.31 \\
\hline $3 g$ & 60 & dysplasia & 64.44 & 93.33 & 28.24 & 48.44 \\
\hline $3 \mathrm{~h}$ & 13 & dysplasia & 17.89 & 35.67 & 60.95 & 79.69 \\
\hline $3 \mathbf{i}$ & 17 & dysplasia & 84.44 & 93.33 & 53.51 & 64.06 \\
\hline $3 \mathbf{j}$ & 7 & dysplasia & 33.33 & 37.78 & 74.33 & 82.81 \\
\hline $3 k$ & 0 & ND & 18.89 & 33.33 & 11.89 & 37.50 \\
\hline 31 & 0 & ND & 15.56 & 32.22 & 16.36 & 62.50 \\
\hline $3 m$ & 0 & ND & 65.56 & 82.22 & 13.37 & 31.25 \\
\hline $3 n$ & 10 & dysplasia & 15.56 & 30.00 & 13.37 & 62.50 \\
\hline 30 & 10 & dysplasia & 12.22 & 16.67 & 5.94 & 18.75 \\
\hline $3 p$ & 7 & dysplasia & 11.11 & 17.78 & 25.27 & 21.87 \\
\hline $3 q$ & 10 & dysplasia & 13.23 & 15.36 & 41.62 & 59.37 \\
\hline $3 \mathbf{r}$ & 0 & ND & 15.56 & 51.11 & - & - \\
\hline $3 s$ & 7 & dysplasia & 12.22 & 22.22 & 35.67 & 50.00 \\
\hline $3 t$ & 0 & ND & 17.78 & 41.11 & 5.95 & 0.00 \\
\hline chlorantraniliprole & 100 & ND & - & - & - & - \\
\hline biflenazate & - & - & 85.56 & 95.56 & 61.11 & 65.62 \\
\hline pyridaben & - & - & 61.11 & 71.21 & 97.78 & 98.44 \\
\hline hexythiazox & - & - & 83.33 & 83.33 & 67.19 & 71.11 \\
\hline
\end{tabular}

a "-" means activity is not tested; " "ND" means no dysplasia. The data in bold are used to emphasize that these compounds showed good activity.

The foliar contact activities of the synthesized compounds against two cotton spider mite species ae shown in Table 1, in comparison to biflenazate, pyridaben and hexythiazox. In general, all of the compounds displayed considerable to excellent acaricidal activity against the two kinds of cotton spider mite. Tschimganin analogs with $4-t-\mathrm{Bu}(3 \mathrm{~g})$ and $4-\mathrm{F}(3 \mathrm{~m})$ at the benzene ring exhibited activity against $T$. truncates similar to pyridaben. Compound $3 \mathbf{i}$, with $3-\mathrm{NO}_{2}$ in the benzene ring, exhibited much better activity than pyridaben, and activity similar to biflenazate and hexythiazox. Notably, tschimganin analogs with 4-OMe (3f), 2- $\mathrm{NO}_{2}(3 \mathbf{h}), 3-\mathrm{NO}_{2}(3 \mathbf{i})$ and $4-\mathrm{NO}_{2}(3 \mathbf{j})$ at the benzene ring displayed activity similar to biflenazate and hexythiazox and slightly lower than that of pymetrozine against T. turkestani Ugarov et Nikolski (Table 1). 

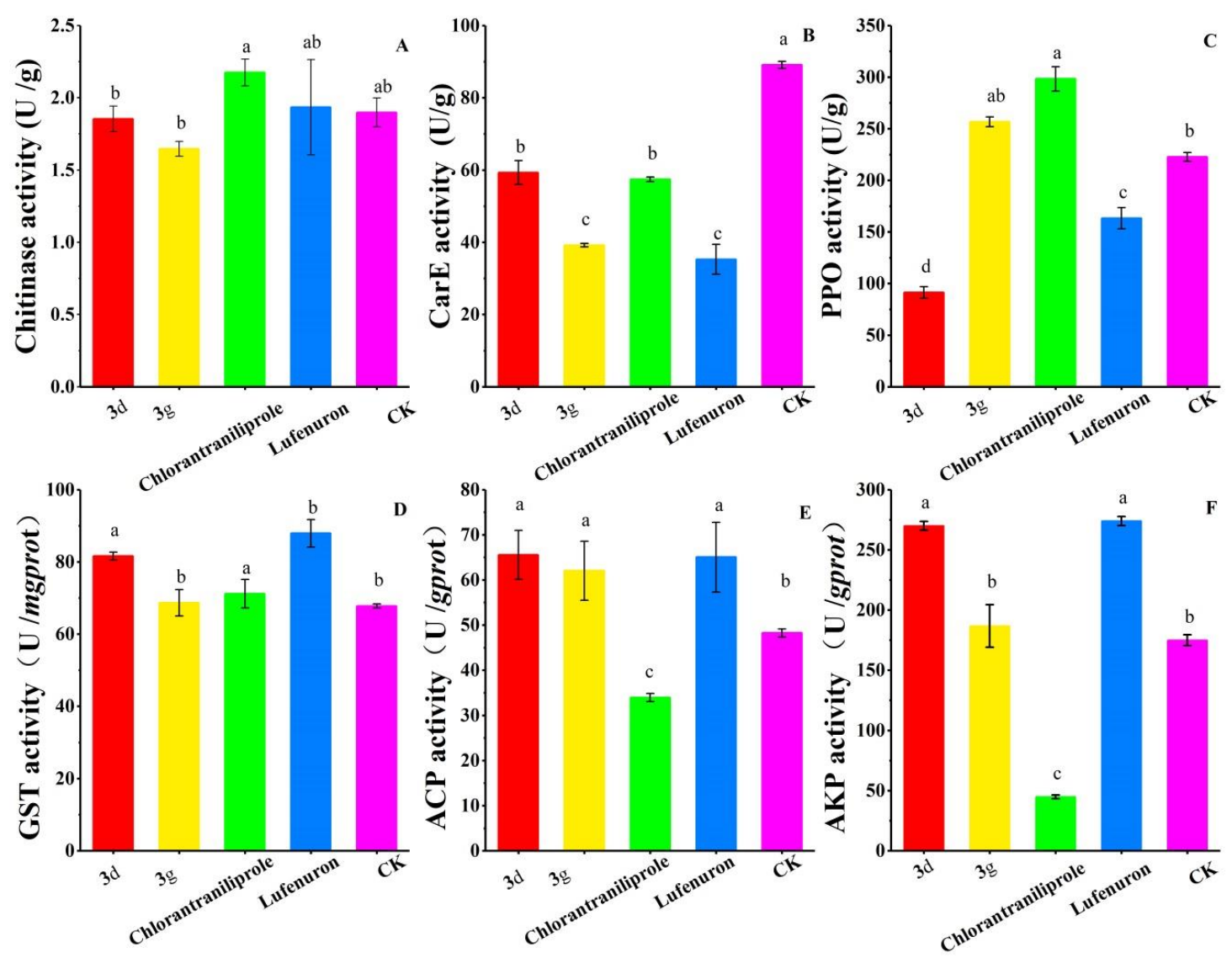

Figure 2. Effect on H. armigera (Hübner) enzyme activity by tschimganin analogs. (A) Chitinase activity; (B) CarE activity; (C) PPO activity; (D) GST activity; (E) ACP activity; (F) AKP activity. ${ }^{\text {a-c }}(p<0.05$; Duncan's test).

However, tschimganin analogs with alkyl, $\mathrm{Cl}, \mathrm{Br}, \mathrm{I}, \mathrm{NH}_{2}, \mathrm{CN}$ and naphthylacetic acid on the benzene ring exhibited much lower activity than control against the two cotton spider mite species. The results indicated that the additive effect of the $t$ - $\mathrm{Bu}$ and $\mathrm{NO}_{2}$ group play an important role in acaricidal activity. In addition, the properties and positions of these substituents on the benzene ring have an important influence on the acaricidal activity of these compounds. Compound $3 \mathbf{i}$, having a $\mathrm{NO}_{2}$ group at the meta position of the benzene ring, exhibited good activity against both cotton spider mite species. $\mathbf{3} \mathbf{h}$ and $\mathbf{3} \mathbf{j}$ only exhibited activity against $T$. turkestani Ugarov et Nikolski; they have a $\mathrm{NO}_{2}$ group at the ortho and para positions of the benzene ring, respectively.

The topomer comparative molecular field analysis (CoMFA) model was optimized. A cross-validation $q^{2}$ value of 0.511 and a non-cross-validation $r^{2}$ value of 0.830 with an optimized component of 3 were obtained, which suggested that the model has good predictive ability $\left(q^{2}>0.5\right)$. The sterically favored and disfavored regions are shown in green and yellow. In the electrostatic field, the positively charged favored regions are shown in blue, and the negatively charged favored regions are shown in red. We chose the molecule $\mathbf{3} \mathbf{j}$, which had the highest activity against $T$. turkestani Ugarov et Nikolski; this makes it is easier to explain the contour map. The activity of the compound is affected by the interaction between the three-dimensional field and the electrostatic field. Compounds $\mathbf{3 g}, \mathbf{3 h}$, $3 \mathbf{i}$, and $3 \mathbf{j}$ all have large-volume groups (Figure $3 \mathrm{~B}$ ). However, there are large red regions on both sides of the benzene ring (Figure $3 \mathrm{C}$ ), indicating that electronegativity was beneficial for acaricidal activity. Therefore, the nitro-substituted compounds (3h, 3i , and $3 \mathbf{j})$ had higher activity than the tert-butyl-substituted compound (3g).

Compounds $\mathbf{3} \mathbf{a}-\mathbf{3} \mathbf{w}$ were evaluated in a series of fungicidal tests in vitro, against a range of phytopathogenic species. The resulting data (Table 2) revealed that these tschimganin analogs displayed 
potential fungicidal activity against kinds of plant fungi, including Colleetotrichum lagenarium (Pass.) Ell. et Halst, Rhizoctonia solani, Fulvia fulva (Cooke) Cif., Pyricularia grisea (Cooke) Sacc., Alternaria alternata Japanese pear pathotype, sunflower sclerotinia rot, and rape sclerotinia rot.
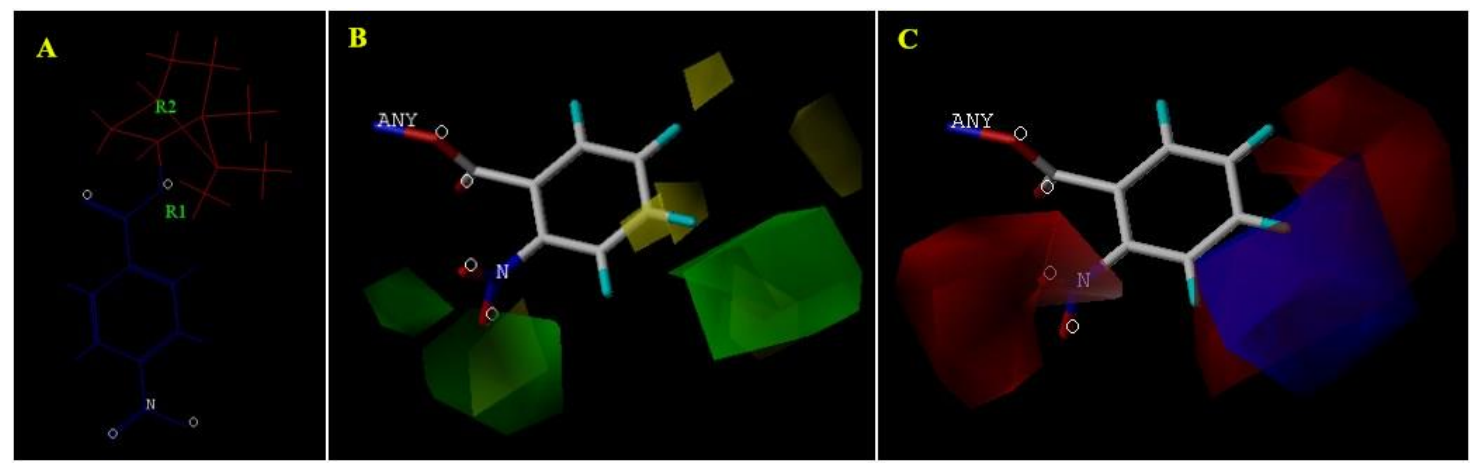

Figure 3. (A) Topomer comparative molecular field analysis (CoMFA) contour maps of 3j; (B) The steric field around R1 of $\mathbf{3} \mathbf{j}$; (C) The electrostatic field around R1 of $\mathbf{3 j}$.

In general, these compounds were highly active against sunflower sclerotinia rot and rape sclerotinia rot, and possessed poor activity against $C$. lagenarium (Pass.) Ell. et Halst and $R$. solani in vitro. Similar to acaricidal activity, the properties of these substituents on the benzene ring have an important influence on the fungicidal activities of these compounds. Compound 3m, which have electron-withdrawing group $\mathrm{F}$ atom, showed moderate fungicidal activity for sunflower sclerotinia rot, rape sclerotinia rot, F. fulva (Cooke) Cif., and A. alternata Japanese pear pathotype.

Table 2. In vitro inhibitory activity against plant pathogens at $50 \mu \mathrm{g} / \mathrm{mL}^{\mathrm{a}}$.

\begin{tabular}{cccccccc}
\hline \multirow{2}{*}{ Compound } & \multicolumn{7}{c}{ Inhibition Ratio (\%) } \\
\cline { 2 - 8 } & $\mathbf{C L}$ & $\mathbf{R S}$ & FF & PG & AK & SSR & RSR \\
\hline 3a & 17.89 & 20.87 & 31.74 & 21.09 & 37.32 & 32.32 & $\mathbf{5 6 . 7 5}$ \\
3b & 0.00 & -4.81 & 4.63 & 32.43 & 11.97 & $\mathbf{5 5 . 4 9}$ & $\mathbf{6 7 . 8 6}$ \\
3c & 0.00 & -9.40 & 8.37 & 23.36 & 37.32 & 39.23 & 36.31 \\
3d & 0.00 & $\mathbf{4 0 . 0 4}$ & 29.64 & 37.19 & 40.85 & 49.39 & 22.62 \\
3e & 0.00 & 10.02 & $\mathbf{5 0 . 4 4}$ & $\mathbf{6 0 . 0 9}$ & $\mathbf{5 7 . 0 4}$ & $\mathbf{4 9 . 5 9}$ & 29.56 \\
3f & 1.42 & -3.76 & 15.38 & 19.73 & 25.35 & 1.22 & 11.71 \\
3g & 0.00 & -2.72 & 3.46 & 31.97 & 22.54 & 42.89 & 4.96 \\
3h & 0.00 & 6.89 & 14.91 & 24.49 & 37.32 & 36.18 & 41.87 \\
3i & 0.00 & -8.98 & 17.02 & 27.44 & 41.55 & 26.63 & $\mathbf{7 3 . 8 1}$ \\
3j & 0.00 & -8.98 & 8.60 & 29.71 & 31.69 & 7.72 & $\mathbf{8 0 . 9 5}$ \\
3k & 19.51 & 4.17 & 22.86 & 24.94 & 40.85 & 13.21 & 18.85 \\
31 & 15.04 & -5.22 & 31.98 & 32.43 & 33.10 & 46.34 & 16.27 \\
3m & 28.46 & -6.06 & 26.60 & 35.83 & 41.55 & $\mathbf{5 7 . 9 3}$ & $\mathbf{5 0 . 6 0}$ \\
3n & 0.00 & 17.32 & 32.21 & 36.51 & 11.97 & 22.97 & 16.27 \\
3o & 0.00 & -10.03 & 15.61 & 24.94 & 26.76 & 17.80 & 8.13 \\
3p & 0.00 & -5.43 & 13.51 & 30.61 & 38.03 & 2.85 & 14.29 \\
3q & 0.00 & 7.30 & 4.63 & 31.29 & $\mathbf{6 3 . 3 8}$ & $\mathbf{5 5 . 4 9}$ & $\mathbf{7 8 . 5 7}$ \\
3r & $\mathbf{7 3 . 1 7}$ & $\mathbf{5 7 . 4 1}$ & $\mathbf{5 0 . 4 4}$ & $\mathbf{5 6 . 4 3}$ & $\mathbf{6 0 . 1 4}$ & $\mathbf{6 5 . 0 4}$ & $\mathbf{8 4 . 3 3}$ \\
3s & 7.11 & 5.22 & 22.86 & 34.92 & 42.25 & 31.10 & 84.13 \\
3t & 6.30 & -2.72 & 17.02 & 33.33 & 7.04 & 3.46 & 4.96 \\
azoxystrobin & $\mathbf{6 6 . 5 4}$ & 18.37 & 55.61 & 73.92 & 61.97 & 89.63 & 90.48 \\
kresoxim-methyl & 0.00 & 1.25 & 25.20 & 21.54 & 19.25 & 2.24 & 75.00 \\
trifloxystrobin & 46.54 & 28.60 & 52.55 & 71.28 & 65.39 & 86.79 & 17.26 \\
\hline
\end{tabular}

a CL: C. lagenarium (Pass.) Ell. et Halst; RS: R. solani; FF: F. fulva (Cooke) Cif.; PG: P. grisea (Cooke) Sacc.; AK: A. alternata Japanese pear pathotype; SSR: Sunflower sclerotinia rot; RSR: Rape sclerotinia rot. The data in bold are used to emphasize that these compounds showed good activity. 
Compound 3e, having a methoxy at the meta position of the benzene ring, exhibited good activity against F. fulva (Cooke) Cif., P. grisea (Cooke) Sacc., A. alternata Japanese pear pathotype and sunflower sclerotinia rot. Compound $\mathbf{3 q}$, possessing an iodine substituent at the para position of the benzene ring, exhibited good activity against $A$. alternata Japanese pear pathotype, sunflower sclerotinia rot, and rape sclerotinia rot. Notably, compound 3r having a 2-hydroxy-3,5-dinitrophenyl moiety displayed a fungicidal spectrum as broad as azoxystrobin against these phytopathogens. We didn't find an explicit relationship between their structures and fungicidal activities for mycelium inhibition.

\section{Materials and Methods}

\subsection{Materials and Measurements}

${ }^{1} \mathrm{H}-\mathrm{NMR}$ and ${ }^{13} \mathrm{C}-\mathrm{NMR}$ spectra were obtained at 400/101 MHz using an Avance III HD 400 spectrometer (Bruker, Zurich, Switzerland) in $\mathrm{CDCl}_{3}$ solution with TMS as the internal standard. Chemical shift values $(\delta)$ were given in parts per million. HRMS data were obtained on a LTQ Orbitrap XL Discovery (Thermo Scientific, Bremen, Germany) instrument. Commercially available compounds were used in this work without further purification. The solvent tetrahydrofuran (THF) and benzene was dried by distillation from sodium and benzophenone. The $\mathrm{CH}_{2} \mathrm{Cl}_{2}$ (DCM) was dried by distillation $\mathrm{CaH}_{2}$. Equipment used included a HVE-50 autoclave (Hirayama Manufacturing Corporation, Tokyo, Japan), a A1000IN Intelligent artificial climate chamber (Conviron, Winnipeg, Manitoba Canada), a DL-CJ-1ND Super Clear Workbanch (Donglian Elactronic \& Technology Development Co., Ltd., Beijing, China). Potato dextrose agar was purchased from Qingdao Hope Bio-Technogy Co., Ltd. (Qingdao, China). Chlorantraniliprole, lufenuron, biflenazate, pyridaben and hexythiazox standards were purchased from J\&K Scientific Ltd. (Beijing, China). Enzyme activity kits were purchased from Nanjing Jiancheng Bioengineering Institute, (Nanjing, China). H. armigera (Hübner), T. truncates, T. turkestani Ugarov et Nikolski were reared in lab. Plant pathogens C. lagenarium (Pass.) Ell. et Halst, R. solani, F. fulva (Cooke) Cif., P. grisea (Cooke) Sacc., A. alternata Japanese pear pathotype, sunflower sclerotinia rot and rape sclerotinia rot were provided by the Key Laboratory at Universities of Xinjiang Uygur Autonomous Region for Oasis Agricultural Pest Management and Plant Protection Resource Utilization Shihezi, China).

\subsection{General Synthetic Method for Compounds 3}

A mixture of substituted benzoic acid 4 (0.01 mol), 1S,2S,4R-fenchyl alcohol (5, $0.011 \mathrm{~mol})$, 4-dimethylaminopyridine (DMAP, $0.002 \mathrm{~mol}$ ), and dichloromethane (DCM, $25 \mathrm{~mL}$ ) was stirred at $0{ }^{\circ} \mathrm{C}$. Then, $N, N^{\prime}$-dicyclohexylcarbodiimide (DCC, $\left.0.008 \mathrm{~mol}\right)$ dissolved in DCM (5 mL) was added dropwise at $0{ }^{\circ} \mathrm{C}$ and then stirred for $10 \mathrm{~h}$ at room temperature. The mixture was filtered, and the filtrate concentrated under reduced pressure. The residue was subjected to silica gel chromatography using PE and EtOAc as eluent to afford the appropriate compound 3.

(1S,2S,4R)-1,7,7-Trimethylbicyclo[2.2.1]heptan-2-yl benzoate (3a): colorless oil, yield 82\%. ${ }^{1} \mathrm{H}-\mathrm{NMR} \delta$ 8.14-8.04 (m, 2H), 7.64-7.54 (m, 1H), 7.51-7.43 (m, 2H), $4.65(\mathrm{~d}, J=1.9 \mathrm{~Hz}, 1 \mathrm{H}), 2.02-1.92(\mathrm{~m}, 1 \mathrm{H})$, 1.85-1.77 (m, 2H), 1.72-1.67 (m, 1H), 1.59-1.49 (m, 1H), $1.28(\mathrm{dd}, J=10.3,1.5 \mathrm{~Hz}, 1 \mathrm{H}), 1.21(\mathrm{~s}, 3 \mathrm{H})$, 1.14 (s, 3H), 0.87 (s, 3H). ${ }^{13} \mathrm{C}-\mathrm{NMR} \delta 166.88,132.76,130.73,86.68,48.65,48.45,41.49,29.78,26.91,25.94$, 20.33, 19.52. HRMS $(m / z)$ calcd. for $\mathrm{C}_{17} \mathrm{H}_{23} \mathrm{O}_{2}{ }^{+}(\mathrm{M}+\mathrm{H})^{+} 259.16926$, found 259.16925 .

(1S,2S,4R)-1,7,7-Trimethylbicyclo[2.2.1]heptan-2-yl 2-methylbenzoate (3b): white powder, m.p. $41.5-42.5{ }^{\circ} \mathrm{C}$, yield 83\%. ${ }^{1} \mathrm{H}-\mathrm{NMR} \delta 7.95(\mathrm{dd}, J=8.1,1.2 \mathrm{~Hz}, 1 \mathrm{H}), 7.40(\mathrm{td}, J=7.5,1.4 \mathrm{~Hz}, 1 \mathrm{H}), 7.26(\mathrm{q}, J=5.2 \mathrm{~Hz}, 2 \mathrm{H})$, $4.60(\mathrm{~d}, J=1.9 \mathrm{~Hz}, 1 \mathrm{H}), 2.62(\mathrm{~s}, 3 \mathrm{H}), 1.94-1.85(\mathrm{~m}, 1 \mathrm{H}), 1.80-1.73(\mathrm{~m}, 2 \mathrm{H}), 1.67(\mathrm{dd}, J=10.3,1.7 \mathrm{~Hz}$, $1 \mathrm{H}), 1.54-1.46(\mathrm{~m}, 1 \mathrm{H}), 1.24(\mathrm{dd}, J=10.3,1.4 \mathrm{~Hz}, 1 \mathrm{H}), 1.21(\mathrm{~s}, 3 \mathrm{H}), 1.16(\mathrm{ddd}, J=6.2,3.3,2.1 \mathrm{~Hz}, 1 \mathrm{H})$, $1.13(\mathrm{~d}, J=4.8 \mathrm{~Hz}, 3 \mathrm{H}), 0.86(\mathrm{~s}, 3 \mathrm{H}) .{ }^{13} \mathrm{C}-\mathrm{NMR} \delta 168.01,140.08,131.74,131.67,130.48,130.23,125.70$, $86.92,48.52,48.48,41.50,39.75,29.76,27.03,25.91,21.93,20.44,19.60$. HRMS $(m / z)$ calcd. for $\mathrm{C}_{18} \mathrm{H}_{25} \mathrm{O}_{2}{ }^{+}$ $(\mathrm{M}+\mathrm{H})^{+}$273.18491, found 273.18500 . 
(1S,2S,4R)-1,7,7-Trimethylbicyclo[2.2.1]heptan-2-yl 4-methylbenzoate (3c): colorless oil, yield 86\%. ${ }^{1} \mathrm{H}-\mathrm{NMR}$ 反 8.01-7.90 (m, 2H), $7.24(\mathrm{~s}, 2 \mathrm{H}), 4.61(\mathrm{~d}, J=1.9 \mathrm{~Hz}, 1 \mathrm{H}), 2.42(\mathrm{~s}, 3 \mathrm{H}), 1.98-1.90(\mathrm{~m}, 1 \mathrm{H}), 1.82-1.74(\mathrm{~m}$, 2H), 1.69-1.64 (m, 1H), $1.50(\mathrm{ddd}, J=12.6,5.8,4.1 \mathrm{~Hz}, 1 \mathrm{H}), 1.28-1.20(\mathrm{~m}, 2 \mathrm{H}), 1.18(\mathrm{~s}, 3 \mathrm{H}), 1.10(\mathrm{~s}, 3 \mathrm{H})$, 0.84 (s, 3H). ${ }^{13} \mathrm{C}-\mathrm{NMR} \delta 171.96,133.82,132.16,131.01,128.68,128.00,127.94,126.21,125.72,125.44$, $124.04,86.74,48.30,48.28,41.28,39.46,39.40,29.60,26.41,25.71,19.94,19.26$. HRMS $(\mathrm{m} / z)$ calcd. for $\mathrm{C}_{18} \mathrm{H}_{25} \mathrm{O}_{2}^{+}(\mathrm{M}+\mathrm{H})^{+}$273.18491, found 273.18494 .

(1S,2S,4R)-1,7,7-Trimethylbicyclo[2.2.1]heptan-2-yl 2-methoxybenzoate (3d): light yellow oil, yield 89\%. ${ }^{1} \mathrm{H}-\mathrm{NMR} \delta 7.86(\mathrm{dd}, J=7.9,1.8 \mathrm{~Hz}, 1 \mathrm{H}), 7.47(\mathrm{ddd}, J=9.1,7.5,1.8 \mathrm{~Hz}, 1 \mathrm{H}), 7.02-6.95(\mathrm{~m}, 2 \mathrm{H}), 4.59(\mathrm{~d}$, $J=1.9 \mathrm{~Hz}, 1 \mathrm{H}), 3.91(\mathrm{~s}, 3 \mathrm{H}), 1.96-1.87(\mathrm{~m}, 1 \mathrm{H}), 1.76(\mathrm{ddq}, J=9.1,6.1,3.0 \mathrm{~Hz}, 2 \mathrm{H}), 1.67-1.62(\mathrm{~m}, 1 \mathrm{H})$, $1.49(\mathrm{tdd}, J=12.5,5.7,4.1 \mathrm{~Hz}, 1 \mathrm{H}), 1.23(\mathrm{dd}, J=10.3,1.4 \mathrm{~Hz}, 1 \mathrm{H}), 1.19(\mathrm{~s}, 3 \mathrm{H}), 1.15(\mathrm{ddd}, J=12.4$, 3.4, $2.1 \mathrm{~Hz}, 1 \mathrm{H}), 0.87$ (s, 3H). ${ }^{13} \mathrm{C}-\mathrm{NMR} \delta 166.49,159.41,133.35,131.67,120.45,120.03,111.98,86.76$, $55.84,48.53,41.45,39.77,29.69,26.89,25.90,20.31,19.52$. HRMS $(m / z)$ calcd. for $\mathrm{C}_{18} \mathrm{H}_{25} \mathrm{O}_{3}{ }^{+}(\mathrm{M}+\mathrm{H})^{+}$ 289.17982 , found 289.17987 .

(1S,2S,4R)-1,7,7-Trimethylbicyclo[2.2.1]heptan-2-yl 3-methoxybenzoate (3e): colorless oil, yield 90\%. ${ }^{1} \mathrm{H}-\mathrm{NMR} \delta 7.66(\mathrm{dd}, J=7.6,0.9 \mathrm{~Hz}, 1 \mathrm{H}), 7.60(\mathrm{dd}, J=2.4,1.4 \mathrm{~Hz}, 1 \mathrm{H}), 7.36(\mathrm{t}, J=7.9 \mathrm{~Hz}, 1 \mathrm{H})$, 7.14-7.07 (m, 1H), $4.61(\mathrm{~d}, J=1.7 \mathrm{~Hz}, 1 \mathrm{H}), 3.86(\mathrm{~s}, 3 \mathrm{H}), 2.00-1.86(\mathrm{~m}, 1 \mathrm{H}), 1.82-1.74(\mathrm{~m}, 2 \mathrm{H}), 1.69-1.65$ $(\mathrm{m}, 1 \mathrm{H}), 1.56-1.48(\mathrm{~m}, 1 \mathrm{H}), 1.25(\mathrm{~d}, J=10.3 \mathrm{~Hz}, 2 \mathrm{H}), 1.19(\mathrm{~s}, 3 \mathrm{H}), 1.11(\mathrm{~s}, 3 \mathrm{H}), 0.85(\mathrm{~s}, 3 \mathrm{H}) .{ }^{13} \mathrm{C}-\mathrm{NMR} \delta$ 166.76, 159.56, 132.04, 129.39, 121.88, 119.01, 114.27, 86.81, 55.40, 48.64, 48.44, 41.47, 39.86, 29.76, 26.90, $20.31,19.51$. HRMS $(m / z)$ calcd. for $\mathrm{C}_{18} \mathrm{H}_{25} \mathrm{O}_{3}{ }^{+}(\mathrm{M}+\mathrm{H})^{+} 289.17982$, found 289.17984 .

(1S,2S,4R)-1,7,7-Trimethylbicyclo[2.2.1]heptan-2-yl 4-methoxybenzoate (3f): colorless oil, yield 86\%. ${ }^{1} \mathrm{H}-\mathrm{NMR} \delta 8.05-8.00(\mathrm{~m}, 2 \mathrm{H}), 6.96-6.92(\mathrm{~m}, 2 \mathrm{H}), 4.59(\mathrm{~d}, J=1.9 \mathrm{~Hz}, 1 \mathrm{H}), 3.87(\mathrm{~s}, 3 \mathrm{H}), 1.93$ (tdd, $J=10.3,6.1,2.9 \mathrm{~Hz}, 1 \mathrm{H}), 1.81-1.74(\mathrm{~m}, 2 \mathrm{H}), 1.66(\mathrm{dt}, J=10.3,2.2 \mathrm{~Hz}, 1 \mathrm{H}), 1.56(\mathrm{~s}, 1 \mathrm{H}), 1.54-1.47(\mathrm{~m}, 1 \mathrm{H})$, $1.24(\mathrm{dd}, J=10.3,1.6 \mathrm{~Hz}, 1 \mathrm{H}), 1.18(\mathrm{~s}, 3 \mathrm{H}), 1.10(\mathrm{~s}, 3 \mathrm{H}), 0.84(\mathrm{~s}, 3 \mathrm{H}) .{ }^{13} \mathrm{C}-\mathrm{NMR} \delta 166.65,163.23,131.51$, $123.18,113.61,86.29,55.44,48.62,48.44,41.48,39.83,29.76,26.92,25.94,20.31,19.52$. HRMS $(\mathrm{m} / \mathrm{z}) \mathrm{calcd}$. for $\mathrm{C}_{18} \mathrm{H}_{25} \mathrm{O}_{3}{ }^{+}(\mathrm{M}+\mathrm{H})^{+} 289.17982$, found 289.17981 .

(1S,2S,4R)-1,7,7-Trimethylbicyclo[2.2.1] heptan-2-yl 4-(tert-butyl)benzoate (3g): white solid, m.p. 74.5-76.3 ${ }^{\circ} \mathrm{C}$, yield 74\%. ${ }^{1} \mathrm{H}-\mathrm{NMR} \delta 8.06-8.02(\mathrm{~m}, 2 \mathrm{H}), 7.52-7.48(\mathrm{~m}, 2 \mathrm{H}), 4.64(\mathrm{~d}, J=1.9 \mathrm{~Hz}, 1 \mathrm{H}), 2.02-1.93(\mathrm{~m}, 1 \mathrm{H})$, 1.85-1.77 (m, 2H), $1.69(\mathrm{dd}, J=10.3,1.7 \mathrm{~Hz}, 1 \mathrm{H}), 1.59-1.51(\mathrm{~m}, 1 \mathrm{H}), 1.37(\mathrm{~s}, 9 \mathrm{H}), 1.27(\mathrm{dd}, J=10.4,1.5 \mathrm{~Hz}$, 1H), 1.21 (s, 3H), 1.13 (s, 3H), $0.87(\mathrm{~s}, 3 \mathrm{H}) .{ }^{13} \mathrm{C}-\mathrm{NMR} \delta 166.87,156.38,129.44,127.97,125.35,86.42,48.64$, $48.46,41.49,39.84,35.07,31.16,29.78,26.91,25.95,20.33,19.51$. HRMS $(m / z)$ calcd. for $\mathrm{C}_{21} \mathrm{H}_{31} \mathrm{O}_{2}{ }^{+}$ $(\mathrm{M}+\mathrm{H})^{+}$315.23186, found 315.23184.

(1S,2S,4R)-1,7,7-Trimethylbicyclo[2.2.1] heptan-2-yl 2-nitrobenzoate (3h): white solid, m.p. 104.3-106.2 ${ }^{\circ} \mathrm{C}$, yield 85\%. ${ }^{1} \mathrm{H}-\mathrm{NMR} \delta 7.89-7.84(\mathrm{~m}, 1 \mathrm{H}), 7.82-7.78(\mathrm{~m}, 1 \mathrm{H}), 7.66(\mathrm{dqd}, J=15.0,7.5,1.6 \mathrm{~Hz}, 2 \mathrm{H}), 4.64(\mathrm{~d}$, $J=2.0 \mathrm{~Hz}, 1 \mathrm{H}), 1.80-1.76(\mathrm{~m}, 1 \mathrm{H}), 1.73-1.63(\mathrm{~m}, 3 \mathrm{H}), 1.53-1.43(\mathrm{~m}, 1 \mathrm{H}), 1.25(\mathrm{dd}, J=10.3,1.5 \mathrm{~Hz}, 1 \mathrm{H})$, $1.21(\mathrm{~s}, 3 \mathrm{H}), 1.15$ (s, 3H), 0.87 (s, 3H). ${ }^{13} \mathrm{C}-\mathrm{NMR} \delta 165.53,148.44,132.58,131.76,130.05,127.61,123.70$, $88.93,48.65,48.33,41.49,39.93,29.66,26.61,25.78,20.21,19.41$. HRMS (m/z) calcd. for $\mathrm{C}_{17} \mathrm{H}_{22} \mathrm{NO}_{4}{ }^{+}$ $(\mathrm{M}+\mathrm{H})^{+}$304.15433, found 304.15475.

(1S,2S,4R)-1,7,7-Trimethylbicyclo[2.2.1]heptan-2-yl 3-nitrobenzoate (3i): white powder, m.p. 84.0-85.0 ${ }^{\circ} \mathrm{C}$, yield 91\%. ${ }^{1} \mathrm{H}-\mathrm{NMR} \delta 8.88(\mathrm{dd}, J=2.8,1.1 \mathrm{~Hz}, 1 \mathrm{H}), 8.48-8.37(\mathrm{~m}, 2 \mathrm{H}), 7.73-7.66(\mathrm{~m}, 1 \mathrm{H}), 4.68(\mathrm{~d}$, $J=1.9 \mathrm{~Hz}, 1 \mathrm{H}), 1.99-1.88(\mathrm{~m}, 1 \mathrm{H}), 1.82(\mathrm{tq}, J=9.0,2.9 \mathrm{~Hz}, 2 \mathrm{H}), 1.70(\mathrm{ddd} J=10.4,4.0,2.3 \mathrm{~Hz}, 1 \mathrm{H})$, 1.64-1.51 (m, 1H), 1.33-1.28 (m, 1H), 1.21 (s, 3H), 1.14 (s, 3H), 0.87 (s, 3H). ${ }^{13} \mathrm{C}-\mathrm{NMR} \delta 164.76,148.34$, 135.15, 132.42, 129.67, 127.26, 124.43, 87.88, 48.63, 48.35, 41.44, 39.88, 29.73, 26.87, 25.89, 20.29, 19.48. HRMS $(m / z)$ calcd. for $\mathrm{C}_{17} \mathrm{H}_{22} \mathrm{NO}_{4}{ }^{+}(\mathrm{M}+\mathrm{H})^{+}$304.15433, found 304.15439.

(1S,2S,4R)-1,7,7-Trimethylbicyclo[2.2.1]heptan-2-yl 4-nitrobenzoate (3j): light yellow solid, m.p. 106.5-108.5 ${ }^{\circ} \mathrm{C}$, yield 87\%. ${ }^{1} \mathrm{H}-\mathrm{NMR} \delta 8.35-8.29(\mathrm{~m}, 2 \mathrm{H}), 8.27-8.21(\mathrm{~m}, 2 \mathrm{H}), 4.67(\mathrm{~d}, J=1.9 \mathrm{~Hz}, 1 \mathrm{H}), 1.97-1.86(\mathrm{~m}, 1 \mathrm{H})$, $1.86-1.76(\mathrm{~m}, 2 \mathrm{H}), 1.70(\mathrm{ddd}, J=10.4,4.0,2.3 \mathrm{~Hz}, 1 \mathrm{H}), 1.63-1.51(\mathrm{~m}, 1 \mathrm{H}), 1.30(\mathrm{dd}, J=10.5,1.5 \mathrm{~Hz}, 1 \mathrm{H})$, $1.21(\mathrm{~s}, 3 \mathrm{H}), 1.14(\mathrm{~s}, 3 \mathrm{H}), 0.86$ (s, 3H). ${ }^{13} \mathrm{C}-\mathrm{NMR} \delta 164.95,150.46,136.02,130.58,123.57,87.86,48.64$, 
$48.35,41.42,39.89,29.72,26.86,25.87,20.27,19.46$. HRMS $(m / z)$ calcd. for $\mathrm{C}_{17} \mathrm{H}_{22} \mathrm{NO}_{4}{ }^{+}(\mathrm{M}+\mathrm{H})^{+}$ 304.15433, found 304.15427.

(1S,2S,4R)-1,7,7-Trimethylbicyclo[2.2.1]heptan-2-yl 2-fluorobenzoate (3k): colorless oil, yield 76\%. ${ }^{1} \mathrm{H}-\mathrm{NMR}$ $\delta 7.97(\mathrm{td}, J=7.6,1.8 \mathrm{~Hz}, 1 \mathrm{H}), 7.51(\mathrm{dddd}, J=8.3,7.0,4.8,1.9 \mathrm{~Hz}, 1 \mathrm{H}), 7.21(\mathrm{td}, J=7.7,1.0 \mathrm{~Hz}, 1 \mathrm{H})$, $7.14(\mathrm{ddd}, J=10.8,8.3,0.9 \mathrm{~Hz}, 1 \mathrm{H}), 4.63(\mathrm{~d}, J=1.8 \mathrm{~Hz}, 1 \mathrm{H}), 1.98-1.89(\mathrm{~m}, 1 \mathrm{H}), 1.77(\mathrm{dd}, J=12.4,6.0 \mathrm{~Hz}$, 2H), 1.69-1.63 (m, 1H), 1.55-1.45 (m, 1H), $1.25(\mathrm{dd}, J=10.3,1.4 \mathrm{~Hz}, 1 \mathrm{H}), 1.19(\mathrm{~s}, 3 \mathrm{H}), 1.12(\mathrm{~s}, 3 \mathrm{H})$, $\left.0.86(\mathrm{~s}, 3 \mathrm{H}) .{ }^{13} \mathrm{C}-\mathrm{NMR} \delta 164.89,163.33,160.74,134.25,132.13,123.92,119.13,117.14,116.92\right), 87.57$, $48.52,48.47,41.43,39.76,29.72,26.83,25.87,20.27,19.46$. HRMS $(m / z)$ calcd. for $\mathrm{C}_{17} \mathrm{H}_{22} \mathrm{FO}_{2}{ }^{+}(\mathrm{M}+\mathrm{H})^{+}$ 277.15983 , found 277.15973 .

(1S,2S,4R)-1,7,7-Trimethylbicyclo[2.2.1] heptan-2-yl 3-fluorobenzoate (31): colorless oil, yield 77\%. ${ }^{1} \mathrm{H}-\mathrm{NMR}$ $\delta 7.69-7.64(\mathrm{~m}, 1 \mathrm{H}), 7.60(\mathrm{dd}, J=2.5,1.5 \mathrm{~Hz}, 1 \mathrm{H}), 7.36(\mathrm{t}, J=7.9 \mathrm{~Hz}, 1 \mathrm{H}), 7.10(\mathrm{ddd}, J=8.3,2.7,0.9 \mathrm{~Hz}$, $1 \mathrm{H}), 4.61(\mathrm{~d}, J=1.9 \mathrm{~Hz}, 1 \mathrm{H}), 1.98-1.89(\mathrm{~m}, 1 \mathrm{H}), 1.82-1.74(\mathrm{~m}, 2 \mathrm{H}), 1.69-1.64(\mathrm{~m}, 1 \mathrm{H}), 1.52(\mathrm{tdd}, J=12.5$, 5.8, $4.1 \mathrm{~Hz}, 1 \mathrm{H}), 1.30-1.19(\mathrm{~m}, 2 \mathrm{H}), 1.19(\mathrm{~s}, 3 \mathrm{H}), 1.11(\mathrm{~s}, 3 \mathrm{H}), 0.85(\mathrm{~s}, 3 \mathrm{H}) .{ }^{13} \mathrm{C}-\mathrm{NMR} \delta 166.78,159.58$, $132.05,129.41,121.89,119.02,114.28,86.82,55.41,48.64,48.44,41.47,39.86,29.77,26.91,25.93,20.32$, 19.51. HRMS $(m / z)$ calcd. for $\mathrm{C}_{17} \mathrm{H}_{22} \mathrm{FO}_{2}{ }^{+}(\mathrm{M}+\mathrm{H})^{+}$277.15983, found 277.15985.

(1S,2S,4R)-1,7,7-Trimethylbicyclo[2.2.1]heptan-2-yl 4-fluorobenzoate (3m): colorless viscous liquid, yield 74\%. ${ }^{1} \mathrm{H}-\mathrm{NMR} \delta 8.14-8.05(\mathrm{~m}, 2 \mathrm{H}), 7.18-7.11(\mathrm{~m}, 2 \mathrm{H}), 4.63(\mathrm{~d}, J=1.9 \mathrm{~Hz}, 1 \mathrm{H}), 1.98-1.88(\mathrm{~m}, 1 \mathrm{H}), 1.80(\mathrm{tt}$, $J=9.1,3.0 \mathrm{~Hz}, 2 \mathrm{H}), 1.71-1.66(\mathrm{~m}, 1 \mathrm{H}), 1.54(\mathrm{ddt}, J=12.6,5.7,4.0 \mathrm{~Hz}, 1 \mathrm{H}), 1.28(\mathrm{dd}, J=10.4,1.5 \mathrm{~Hz}, 1 \mathrm{H})$, $1.20(\mathrm{~s}, 3 \mathrm{H}), 1.13(\mathrm{~s}, 3 \mathrm{H}), 0.86(\mathrm{~s}, 3 \mathrm{H}) .{ }^{13} \mathrm{C}-\mathrm{NMR} \delta 166.93,165.90,164.41,132.04,131.95,126.95,126.92$, $115.59,115.37,86.84,48.61,48.40,41.45,39.83,29.74,26.89,25.91,20.29,19.49$. HRMS $(\mathrm{m} / z)$ calcd. for $\mathrm{C}_{17} \mathrm{H}_{22} \mathrm{FO}_{2}{ }^{+}(\mathrm{M}+\mathrm{H})^{+}$277.15983, found 277.15979.

(1S,2S,4R)-1,7,7-Trimethylbicyclo[2.2.1]heptan-2-yl 2-chlorobenzoate (3n): white solid, m.p. 63.1-63.4 ${ }^{\circ} \mathrm{C}$, yield 78\%. ${ }^{1} \mathrm{H}-\mathrm{NMR} \delta$ 7.90-7.85 $(\mathrm{m}, 1 \mathrm{H}), 7.50-7.40(\mathrm{~m}, 2 \mathrm{H}), 7.37-7.31(\mathrm{~m}, 1 \mathrm{H}), 4.65(\mathrm{~d}, J=2.0 \mathrm{~Hz}, 1 \mathrm{H})$, 1.97-1.87 (m, 1H), 1.82-1.72 (m, 2H), 1.71-1.65 (m, 1H), $1.52(\mathrm{tdd}, J=12.5,5.8,4.1 \mathrm{~Hz}, 1 \mathrm{H}), 1.27(\mathrm{dd}$, $J=10.3,1.5 \mathrm{~Hz}, 1 \mathrm{H}), 1.23(\mathrm{~s}, 3 \mathrm{H}), 1.16(\mathrm{~s}, 3 \mathrm{H}), 0.90(\mathrm{~s}, 3 \mathrm{H}) .{ }^{13} \mathrm{C}-\mathrm{NMR} \delta 166.16,133.59,132.33,131.41$, 131.10, 130.65, 126.56, 88.01, 48.49, 48.44, 41.46, 39.77, 29.71, 26.91, 25.85, 20.38, 19.56. HRMS $(\mathrm{m} / \mathrm{z})$ calcd. for $\mathrm{C}_{17} \mathrm{H}_{22} \mathrm{ClO}_{2}{ }^{+}(\mathrm{M}+\mathrm{H})^{+} 293.13028$, found 293.13025 .

(1S,2S,4R)-1,7,7-trimethylbicyclo[2.2.1] heptan-2-yl 4-chlorobenzoate (3o): colorless oil, yield $75 \% .{ }^{1} \mathrm{H}$ - NMR ס 8.04-7.95 (m, 2H), 7.46-7.39 (m, 2H), $4.61(\mathrm{~d}, J=1.9 \mathrm{~Hz}, 1 \mathrm{H}), 1.94-1.86(\mathrm{~m}, 1 \mathrm{H}), 1.78(\mathrm{ddt}, J=9.0,6.0$, $2.9 \mathrm{~Hz}, 2 \mathrm{H}), 1.67(\mathrm{dd}, J=10.4,1.7 \mathrm{~Hz}, 1 \mathrm{H}), 1.54-1.47(\mathrm{~m}, 1 \mathrm{H}), 1.28-1.19(\mathrm{~m}, 2 \mathrm{H}), 1.18(\mathrm{~s}, 3 \mathrm{H}), 1.10(\mathrm{~s}, 3 \mathrm{H})$, 0.83 (s, 3H). ${ }^{13} \mathrm{C}-\mathrm{NMR} \delta$ 166.05, 139.22, 130.91, 129.15 (s), 128.74, 87.01, 48.63, 48.40), 41.46, 39.86, 29.75, $26.89,25.91,20.29,19.49$. HRMS $(m / z)$ calcd. for $\mathrm{C}_{17} \mathrm{H}_{22} \mathrm{ClO}_{2}{ }^{+}(\mathrm{M}+\mathrm{H})^{+} 293.13028$, found 293.13031.

(1S,2S,4R)-1,7,7-Trimethylbicyclo[2.2.1]heptan-2-yl 4-bromobenzoate (3p): light yellow oil, yield 79\%. ${ }^{1} \mathrm{H}-\mathrm{NMR} \delta$ 7.96-7.88 (m, 2H), 7.63-7.54 (m, 2H), $4.61(\mathrm{~d}, J=1.9 \mathrm{~Hz}, 1 \mathrm{H}), 1.94-1.85(\mathrm{~m}, 1 \mathrm{H}), 1.78(\mathrm{ddt}$, $J=9.1,5.8,3.0 \mathrm{~Hz}, 2 \mathrm{H}), 1.66(\mathrm{dd}, J=10.4,1.7 \mathrm{~Hz}, 1 \mathrm{H}), 1.53-1.47(\mathrm{~m}, 1 \mathrm{H}), 1.28-1.19(\mathrm{~m}, 2 \mathrm{H}), 1.18(\mathrm{~s}, 3 \mathrm{H})$, $1.10(\mathrm{~s}, 3 \mathrm{H}), 0.83(\mathrm{~s}, 3 \mathrm{H}) .{ }^{13} \mathrm{C}-\mathrm{NMR} \delta 166.18,131.74,131.06,129.60,127.86,87.04,48.63,48.40,41.46$, 39.86, 29.75, 26.89, 25.90, 20.28, 19.49. HRMS $(m / z)$ calcd. for $\mathrm{C}_{17} \mathrm{H}_{22} \mathrm{BrO}_{2}{ }^{+}(\mathrm{M}+\mathrm{H})^{+}$337.07977, found 337.08023 .

(1S,2S,4R)-1,7,7-Trimethylbicyclo[2.2.1] heptan-2-yl 4-iodobenzoate (3q): white solid, m.p. 112.9-114.5 ${ }^{\circ} \mathrm{C}$, yield $85 \% .{ }^{1} \mathrm{H}-\mathrm{NMR} \delta 8.02(\mathrm{dd}, J=7.9,1.1 \mathrm{~Hz}, 1 \mathrm{H}), 7.83(\mathrm{dd}, J=7.8,1.7 \mathrm{~Hz}, 1 \mathrm{H}), 7.43(\mathrm{td}, J=7.6,1.2 \mathrm{~Hz}$, $1 \mathrm{H}), 7.21-7.13(\mathrm{~m}, 1 \mathrm{H}), 4.66(\mathrm{~d}, J=1.9 \mathrm{~Hz}, 1 \mathrm{H}), 1.95-1.86(\mathrm{~m}, 1 \mathrm{H}), 1.82-1.73(\mathrm{~m}, 2 \mathrm{H}), 1.72-1.66(\mathrm{~m}, 1 \mathrm{H})$, $1.52(\mathrm{tdd}, J=12.5,5.8,4.1 \mathrm{~Hz}, 1 \mathrm{H}), 1.27(\mathrm{dd}, J=10.3,1.5 \mathrm{~Hz}, 1 \mathrm{H}), 1.24(\mathrm{~s}, 3 \mathrm{H}), 1.18(\mathrm{~s}, 3 \mathrm{H}), 0.91(\mathrm{~s}, 3 \mathrm{H})$. ${ }^{13} \mathrm{C}-\mathrm{NMR} \delta 166.86,141.35,135.66,132.41,130.69,127.88,94.13,87.81,48.55,48.42,41.51,39.85,29.76$, $26.88,25.87,20.47,19.69$. HRMS $(m / z)$ calcd. for $\mathrm{C}_{17} \mathrm{H}_{22} \mathrm{IO}_{2}{ }^{+}(\mathrm{M}+\mathrm{H})^{+} 385.06590$, found 385.06583 .

(1S,2S,4R)-1,7,7-Trimethylbicyclo[2.2.1]heptan-2-yl 2-hydroxy-3,5-dinitrobenzoate (3r): yellow powder, m.p. 108.1-109.2 ${ }^{\circ} \mathrm{C}$, yield 79\%. ${ }^{1} \mathrm{H}-\mathrm{NMR} \delta 12.90(\mathrm{~s}, 1 \mathrm{H}), 9.06(\mathrm{~d}, J=2.8 \mathrm{~Hz}, 1 \mathrm{H}), 8.98(\mathrm{~d}, J=2.9 \mathrm{~Hz}, 1 \mathrm{H})$, $4.75(\mathrm{~d}, J=1.9 \mathrm{~Hz}, 1 \mathrm{H}), 1.87(\mathrm{dd}, J=3.7,2.2 \mathrm{~Hz}, 2 \mathrm{H}), 1.65-1.55(\mathrm{~m}, 2 \mathrm{H}), 1.39-1.33(\mathrm{~m}, 2 \mathrm{H}), 1.23(\mathrm{~s}, 3 \mathrm{H})$, 
1.17 (s, 3H), 0.89 (s, 3H). ${ }^{13}$ C-NMR $\delta 168.35,159.92,129.55,126.55,90.53,48.70,48.23,41.40,40.09,29.59$, $26.81,25.78,20.32$, 19.45. HRMS $(m / z)$ calcd. for $\mathrm{C}_{17} \mathrm{H}_{19} \mathrm{~N}_{2} \mathrm{O}_{7}{ }^{-}(\mathrm{M}-\mathrm{H})^{-} 363.11977$, found 363.11548 .

(1S,2S,4R)-1,7,7-Trimethylbicyclo[2.2.1]heptan-2-yl 4-cyanobenzoate (3s): white solid, m.p. 115.2-117.4 ${ }^{\circ} \mathrm{C}$, yield 80\%. ${ }^{1} \mathrm{H}-\mathrm{NMR} \delta 8.19-8.15(\mathrm{~m}, 2 \mathrm{H}), 7.80-7.75(\mathrm{~m}, 2 \mathrm{H}), 4.66(\mathrm{~d}, J=1.9 \mathrm{~Hz}, 1 \mathrm{H}), 1.95-1.86(\mathrm{~m}, 1 \mathrm{H})$, $1.84-1.75(\mathrm{~m}, 2 \mathrm{H}), 1.72-1.66(\mathrm{~m}, 1 \mathrm{H}), 1.55(\mathrm{tdd}, J=12.6,5.7,4.1 \mathrm{~Hz}, 1 \mathrm{H}), 1.29(\mathrm{dd}, J=10.5,1.6 \mathrm{~Hz}, 1 \mathrm{H})$, $1.20(\mathrm{~s}, 3 \mathrm{H}), 1.13(\mathrm{~s}, 3 \mathrm{H}), 0.85$ (s, 3H). ${ }^{13} \mathrm{C}-\mathrm{NMR} \delta 165.21,134.47,132.27,130.00,118.03,116.25,87.70$, $48.63,48.35,41.42,39.89,39.88,29.73,26.86,25.87,20.27,19.47$. HRMS $(m / z)$ calcd. for $\mathrm{C}_{18} \mathrm{H}_{22} \mathrm{NO}_{2}{ }^{+}$ $(\mathrm{M}+\mathrm{H})^{+}$284.16451, found 284.16534.

(1S,2S,4R)-1,7,7-Trimethylbicyclo[2.2.1]heptan-2-yl 2-(naphthalen-1-yl)acetate (3t): colorless oil, yield 94\%. ${ }^{1} \mathrm{H}-\mathrm{NMR} \delta 8.08-7.99(\mathrm{~m}, 1 \mathrm{H}), 7.84(\mathrm{t}, J=8.8 \mathrm{~Hz}, 1 \mathrm{H}), 7.81-7.74(\mathrm{~m}, 1 \mathrm{H}), 7.56-7.45(\mathrm{~m}, 2 \mathrm{H}), 7.44-7.39(\mathrm{~m}$, $2 \mathrm{H}), 4.33(\mathrm{~s}, 1 \mathrm{H}), 4.10(\mathrm{~d}, J=1.5 \mathrm{~Hz}, 2 \mathrm{H}), 1.63(\mathrm{~d}, J=1.3 \mathrm{~Hz}, 1 \mathrm{H}), 1.59-1.40(\mathrm{~m}, 4 \mathrm{H}), 1.35(\mathrm{tt}, J=10.9$, $5.8 \mathrm{~Hz}, 1 \mathrm{H}), 1.10(\mathrm{~d}, J=10.2 \mathrm{~Hz}, 1 \mathrm{H}), 1.02(\mathrm{~d}, J=2.4 \mathrm{~Hz}, 3 \mathrm{H}), 0.89(\mathrm{~d}, J=2.3 \mathrm{~Hz}, 3 \mathrm{H}), 0.57(\mathrm{~d}, J=2.2 \mathrm{~Hz}$, 3H). ${ }^{13}$ C-NMR $\delta 166.98,143.41,129.58,129.09,128.01,86.45,48.64,48.45,41.48,39.85,29.77,26.91,25.94$, 21.66, 20.31, 19.51. HRMS $(m / z)$ calcd. for $\mathrm{C}_{22} \mathrm{H}_{27} \mathrm{O}_{2}{ }^{+}(\mathrm{M}+\mathrm{H})^{+}$323.20056, found 323.20062.

\subsection{Insecticidal Activity}

All the insecticidal bioassays were performed using representative test organisms prepared in the laboratory. The bioassay was performed at $25 \pm 1{ }^{\circ} \mathrm{C}$. All the compounds were dissolved in DMF and diluted with $0.05 \%$ Triton X-100 to obtain a series of concentrations. The insecticidal activities of the compounds against $H$. armigera (Hübner), T. truncatus and T. turkestani Ugarov et Nikolski were tested according to previously reported procedures [18]. Each administration was repeated three times. Chlorantraniliprole, biflenazate, pyridaben and hexythiazox purchased from J\&K Scientific Ltd., were used as a control and used in the same way.

\subsection{Enzyme Activity}

The enzyme activity was measured according to the assay kit instructions provided by the manufacturer (Nanjing Jiancheng Bioengineering Institute). Sample preparation and purification were performed as follows: $0.1 \mathrm{~g}$ of $\mathrm{H}$. armigera tissues were accurately weighed, a $1 \mathrm{~mL}$ extracted solution and homogenate added under an ice bath, and the supernatant placed on the ice after centrifugation (chitinase: 12,000 rmp, $4{ }^{\circ} \mathrm{C}$, centrifugation for $20 \mathrm{~min}$; carboxylic acid esterase: $12,000 \mathrm{rmp}, 4{ }^{\circ} \mathrm{C}$, centrifugation for $30 \mathrm{~min}$; glutathione $S$-transferase: $10,000 \mathrm{rmp}, 4^{\circ} \mathrm{C}$ centrifugation for $10 \mathrm{~min}$; polyphenol oxidase: $8000 \mathrm{rmp}$, room temperature centrifugation $10 \mathrm{~min}$ ). The sample pretreatment of acid phosphatase and alkaline phosphatase was as follows: $0.1 \mathrm{~g}$ of $H$. armigera tissues were accurately weighed, $0.9 \mathrm{~mL}$ of physiological saline and homogenate was added under an ice bath, then the mixture was centrifugation at $2500 \mathrm{rpm}$ for $10 \mathrm{~min}$, and the supernatant was determined.

\subsection{Antifungal Activity}

The antifungal activities of the synthesized compounds were performed according to previously reported procedures [19]. The fungicidal activities of the target compounds against $C$. lagenarium (Pass.) Ell. et Halst, R. solani, F. fulva (Cooke) Cif, P. grisea (Cooke) Sacc, A. alternata Japanese pear pathotype, sunflower sclerotinia rot and rape sclerotinia rot were evaluated using a mycelium growth rate test. Azoxystrobin, kresoxim-methyl, and trifloxystrobin purchased from J\&K Scientific Ltd., were used as controls, and were utilized in the same way. The relative inhibition ratio (\%) was calculated:

The relative inhibition ratio $(\%)=\frac{\text { colony diameter of control }- \text { colony diameter of treated }}{\text { colony diameter of control mycelial disk diameter }} \times 100 \%$ 


\subsection{Quantitative Structure-Activity Relationship (QSAR) Analyses}

Three-dimensional QSAR analyses were performed to predict the favorable and unfavorable moieties for improved bioactivity using the CoMFA models in the SYBYL7.3 software. CoMFA models were generated using the Sybyl 7.3 package (Certara, Princeton, NJ, USA) on a Linux system. In total, 20 compounds obtained from synthesis were used to create a data set in which the bioactivity of all compounds were determined against $T$. turkestani Ugarov et Nikolski. Three-dimensional molecular structures were built using the SKETCH module in Sybyl 7.3, while structural energy minimization was performed with the Tripos force field until a gradient convergence of $0.05 \mathrm{kcal} /(\mathrm{mol} \mathrm{A})$ was achieved. Gasteiger-Hückel charges were calculated and used to construct the CoMFA models [20].

\section{Conclusions}

In summary, we have designed and synthesized a novel series of the title compounds based on the active structure of tschimganin, and assayed their in vitro insecticidal, acaricidal and fungicidal activity against seven fungi. Compounds $\mathbf{3} \mathbf{d}$ and $\mathbf{3 g}$, with electron donating groups, showed better inhibitory activity and growth inhibition activity to $H$. armigera (Hübner). Morphological and enzymatic assays demonstrated that the compounds $\mathbf{3} \mathbf{d}$ and $\mathbf{3 g}$ could effectively inhibit the growth and development of cotton bollworm. The antifungal evaluation showed that the properties and positions of these substituents on the benzene ring have an important influence on the acaricidal activity of tschimganin analogs. The 3D-QSAR model for the compounds against T. turkestani Ugarov et Nikolski indicated that the electronegativity was beneficial for acaricidal activity. Notably, compound $3 \mathbf{r}$ having a 2-hydroxy-3,5-dinitrophenyl moiety displayed a fungicidal spectrum as broad as that of azoxystrobin against these phytopathogens. All results suggested these compounds have potential to be used as bifunctional agrochemicals. Further studies on structural optimization are in progress at our laboratory.

Supplementary Materials: Supplementary data (i.e., the ${ }^{1} \mathrm{H}-,{ }^{13} \mathrm{C}-\mathrm{NMR}$ and HRMS spectra of all compounds mentioned in this article) are available online.

Author Contributions: X.H. and J.Z. conceived and designed the research. Y.Z., C.W., and F.X. performed the experiments. Y.Z. and X.H. analyzed the data. J.Z. and X.H. wrote the paper. K.S. revised the manuscript.

Funding: This research was supported financially by the State Key Laboratory of the Discovery and Development of Novel Pesticide (Shenyang Sinochem Agrochemicals R\&D Co., Ltd.) (No. 2017NYRD03).

Conflicts of Interest: The authors declare no conflict of interest.

\section{References}

1. Li, L.; Li, Z.; Wang, K.J.; Liu, Y.X.; Li, Y.Q.; Wang, Q.M. Synthesis and antiviral, insecticidal, and fungicidal activities of gossypol derivatives containing alkylimine, oxime or hydrazine moiety. Bioorg. Med. Chem. 2016, 24, 474-483. [CrossRef] [PubMed]

2. Su, J. Dictionary of Chinese Traditional Medicines; Shanghai People's Press: Shanghai, China, 1998; pp. $1186-1187$.

3. Zhou, Y.T.; Xin, F.; Zhang, G.Q.; Qu, H.X.; Yang, D.S.; Han, X.Q. Recent Advances on Bioactive Constituents in Ferula. Drug Dev. Res. 2017, 78, 321-331. [CrossRef] [PubMed]

4. Ahmed, A.A.; Hegazy, M.E.; Zellagui, A.; Rhouati, S.; Mohamed, T.A.; Sayed, A.A.; Mohamed, A.A.; Shinji, O.; Toshifumi, H. Ferulsinaic acid, a sesquiterpene coumarin with a rare carbon skeleton from Ferula species. Phytochemistry 2007, 68, 680-686. [CrossRef] [PubMed]

5. Li, G.Z.; Wang, J.C.; Li, X.J.; Cao, L.; Gao, L.; Lv, N.; Si, J.Y. An unusual sesquiterpene coumarin from the seeds of Ferula sinkiangensis. J. Asian Nat. Prod. Res. 2016, 18, 891-896. [CrossRef] [PubMed]

6. Appendino, G.; Maxia, L.; Bascope, M.; Houghton, P.J.; Gonzalo, S.D.; Munoz, E.; Sterner, O. A Meroterpenoid NF-кB Inhibitor and Drimane Sesquiterpenoids from Asafetida. J. Nat. Prod. 2006, 69, 1101-1104. [CrossRef] [PubMed]

7. Huang, J.; Han, H.Y.; Li, G.Y.; Wang, H.Y.; Zhang, C.; Zhang, K.; Tan, Y.; Li, P.Y.; Wang, J.H. Two new terpenoid benzoates with antitumor activity from the roots of Ferula dissecta. J. Asian Nat. Prod. Res. 2013, 15, 1100-1106. [CrossRef] [PubMed] 
8. Xin, Y.C.; Li, N.; Zhou, D.; Chen, G.; Jiao, K.; Wang, W.L.; Si, Y.Y.; Hou, Y. Sesquiterpene Coumarins from Ferula sinkiangensis Act as Neuroinflammation Inhibitors. Planta Med. 2017, 83, 135-142. [CrossRef]

9. Alam, M.; Khan, A.; Wadood, A.; Ayesha, K.; Bashir, S.; Aman, A.; Jan, A.K.; Rauf, A.; Ahmad, B.; Khan, A.R.; et al. Bioassay-Guided Isolation of Sesquiterpene Coumarins from Ferula narthex Bioss: A New Anticancer Agent. Front. Pharmacol. 2016, 7, 16. [CrossRef]

10. Li, X.J.; Jiang, L.; Da, P. Preparation and investigation of the pharmacodynamics of effective antiulcerative composition in ferula sinkiangensis K. M. Shen. Mod. Chin. Med. 2007, 9, 8-10. (In Chinese) [CrossRef] [PubMed]

11. Iranshahy, M.; Iranshahi, M. Traditional uses, phytochemistry and pharmacology of asafoetida (Ferula assa-foetida oleo-gum-resin)—A review. J. Ethnopharmacol. 2011, 134, 1-10. [CrossRef] [PubMed]

12. Dastan, D.; Salehi, P.; Aliahmadi, A.; Gohari, A.R.; Maroofi, H.; Ardalan, A. New coumarin derivatives from Ferula pseudalliacea with antibacterial activity. Nat. Prod. Res. 2016, 30, 2747-2753. [CrossRef] [PubMed]

13. Mansour, Z.; Insaf, F.; Aymen, J.; Joseph, C.; Jalloul, B.; Hichem, B.J. Chemical Composition and In Vitro Evaluation of Antimicrobial, Antioxidant and Antigerminative Properties of the Seed Oil from the Tunisian Endemic Ferula tunetana POMEL $_{\text {ex B }}$ ATT. Chem. Biodivers. 2017, 14, e1600116. [CrossRef]

14. Samaneh, F.; Habib, A.; Ayatallah, S. Phytochemical and Acaricidal Study of the Galbanum, Ferula gumosa Boiss. (Apiaceae) Essential Oil against Tetranychus urticae Koch (Tetranychidae). J. Essent. Oil Bear. Plants 2017, 20, 185-195. [CrossRef]

15. Adnan, A.; Emmy, T.; Paul, C.; Louis, M.; Vassiliki, E.; Sandra, A.; Luc, P. Antiprotozoal and Antiglycation Activities of Sesquiterpene Coumarins from Ferula narthex Exudate. Molecules 2016, 21, 1287. [CrossRef]

16. Nazrullaev, S.; Saidkhodzhaev, S.; Akhmedkhodzhaeva, A.I.; Syrov, V.N.; Rasulev, B.F.; Khushbaktova, Z.A. Estrogen activity of terpenoids from plants of the genus Ferula. Chem. Nat. Compd. 2008, 44, 572-577. [CrossRef]

17. Trusheva, B.; Todorov, I.; Ninova, M.; Najdenski, H.; Daneshmand, A.; Bankova, V. Antibacterial mono- and sesquiterpene esters of benzoic acids from Iranian propolis. Chem. Cent. J. 2010, 4, 8. [CrossRef] [PubMed]

18. Yang, Y.; Liu, Y.X.; Song, H.J.; Li, Y.Q.; Wang, Q.M. Additive effects on the improvement of insecticidal activity: Design, synthesis, and insecticidal activity of novel pymetrozine derivatives. Bioorg. Med. Chem. 2016, 24, 391-402. [CrossRef] [PubMed]

19. Huo, X.Y.; Guo, L.; Chen, X.F.; Zhou, Y.T.; Zhang, J.; Han, X.Q.; Dai, B. Design, Synthesis, and Antifungal Activity of Novel Aryl-1,2,3-Triazole- $\beta$-Carboline Hybrids. Molecules 2018, 23, 1344. [CrossRef] [PubMed]

20. Du, S.J.; Lu, H.Z.; Yang, D.Y.; Li, H.; Gu, X.L.; Wan, C.; Jia, C.Q.; Wang, M.; Li, X.Y.; Qin, Z.H. Synthesis, Antifungal Activity and QSAR of Some Novel Carboxylic Acid Amides. Molecules 2015, 20, 4071-4087. [CrossRef] [PubMed]

Sample Availability: Samples of the compounds $\mathbf{8 a - 8 t}$ are available from the authors.

(C) 2018 by the authors. Licensee MDPI, Basel, Switzerland. This article is an open access article distributed under the terms and conditions of the Creative Commons Attribution (CC BY) license (http://creativecommons.org/licenses/by/4.0/). 